\title{
Autoimmune Hepatitis: An Update on Current Animal Models
}

\author{
D.P.B. Bogdanos ${ }^{1}$ and U. Christen ${ }^{*, 2}$ \\ ${ }^{1}$ Liver Immunopathology, Institute of Liver Studies, King's College London School of Medicine at King's College \\ London Hospital, London, United Kingdom \\ ${ }_{2}^{2}$ Pharmazentrum Frankfurt, Johann Wolfgang Goethe University / ZAFES, Frankfurt am Main, Germany
}

\begin{abstract}
Autoimmune hepatitis (AIH) is a chronic liver disease of unknown origin characterized serologically by the presence of hyperglobulinemia and high-titre autoantibodies and histologically by interface hepatitis leading to cirrhosis. The immunopathogenesis of AIH is not well understood. Hepatocyte destruction in AIH is likely to originate from an autoimmune attack but what it is responsible for the loss of immunological tolerance to disease-related autoantigens is unknown. In this review, we critically evaluate the current experimental AIH models and discuss their advantages and disadvantages in relation to the human disease.
\end{abstract}

Keywords: Autoimmunity, autoantibodies, cellular immunity, liver disease, pathogenesis.

\section{INTRODUCTION}

Autoimmune hepatitis (AIH) is a chronic progressive liver disease of unknown cause which is characterized by an immune-mediated destruction of hepatocytes leading to cirrhosis and subsequent liver failure $[1,2]$. The disease has a female predominance, occurs in children and adults of all ages and affects different ethnic groups [1]. The prevalence of AIH is $\sim 20$ cases per million persons in Northern Europe and an estimated 100,000 to 200,000 persons are currently affected with this disease in USA [3, 4]. The clinical spectrum of AIH varies and affected cases can present as asymptomatic, with symptoms indistinguishable from those of an acute viral hepatitis or with fulminant hepatic failure [1]. Extrahepatic immune-mediated diseases such as autoimmune thyroiditis, rheumatoid arthritis, ulcerative colitis and type 1 diabetes mellitus are relatively common at presentation and may develop at any time during the course of $\operatorname{AIH}[1,2,5]$. AIH responds well to conventional immunosuppressive treatment consisting of prednisone (or prednisolone) alone or in combination with azathioprine and remission is achieved in approximately $90 \%$ AIH cases $[1,2,5]$. When AIH presents as acute liver failure and does not respond to immunosuppression it may require transplantation [5]. The histological picture of AIH is that of interface hepatitis characterized by mononuclear cells, lymphocytes, plasma cells and macrophages invading the limiting plate and progressing to lobular hepatitis (Fig. 1) [1]. The serological hallmark of the disease is the presence of elevated serum aminotransferase levels, hypergammaglobulinemia and high-titre autoantibodies [1].

Based on distinct autoantibody profiles, AIH is subdivided to type 1 (AIH-1) characterized by seropositivity for anti-nuclear antibody (ANA) and/or smooth muscle antibody (SMA) and type 2 (AIH-2) with seropositivity for anti-liver kidney microsomal type 1 (anti-LKM1) antibodies [1, 2, 6]. No single AIH-1-specific nuclear antigen has been identified

*Address correspondence to this author at the Pharmazentrum Frankfurt, Klinikum der Johann Wolfgang Goethe Universität, Theodor-Stern Kai 7, D-60590 Frankfurt am Main, Germany; Tel: +49-69-6301-83105; Fax: +4969-6301-7663; E-mail: christen@med.uni-frankfurt.de

so far and AIH-related ANA can recognize centromere, histones, double stranded DNA, chromatin, and ribonucleoprotein complexes [7]. SMA in AIH-1 are directed against polymerized filamentous actin and non-actin components [7, 8]. Anti-LKM1 antibodies recognize the microsomal cytochrome P450IID6 (CYP2D6) enzyme. Anti-CYP2D6 antibodies are specifically present in approximately $85 \% \mathrm{AIH}-2$ patients and in a minor proportion (up to $5 \%$ ) of unselected chronic hepatitis C virus infected patients. Asialoglycoprotein receptor (ASGP-R), soluble liver antigen (SLA) and formiminotransferase cyclodeaminase (FTCD) have also been identified as target antigens of autoimmune responses in patients with AIH [7].

Normal

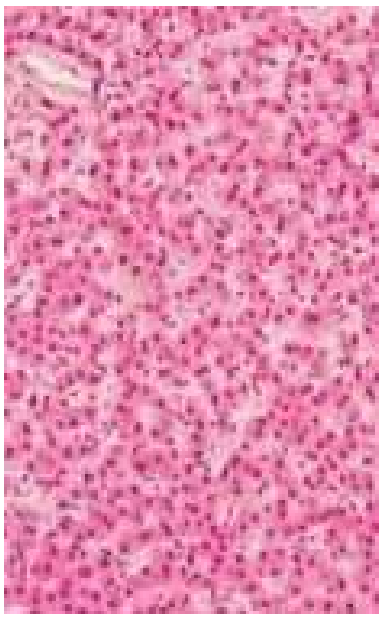

\section{Liver Histology}

Fig. (1). Histological picture details of normal (left) and pathological (a patient with autoimmune hepatitis) liver (right).

Susceptibility to AIH-1 is conferred by the possession of HLA DR3 and DR4 as more than $80 \%$ of AIH-1 cases from USA or northern Europe have HLA DR3, DR4, or DR3 and DR4. AIH-2 is mainly associated with possession of DR7 [2, 9]. 


\section{PATHOGENESIS OF AUTOIMMUNE HEPATITIS}

Histological examination of the liver tissue of patients with AIH reveals a large number of mononuclear cells, macrophages, plasma cells and lymphocytes infiltrating the portal tract and the adjacent parenchyma (Fig. 1). T lymphocytes expressing the $\alpha / \beta \mathrm{T}$ cell receptor predominate and the majority of these cells are positive for the CD4 helper/inducer phenotype and/or the CD8 cytotoxic/suppressor phenotype $[1,10]$. A substantial number of natural killer (NK) cells, natural killer T cells (NKT), macrophages, and B cells can also be found [11].

What promotes the massive inflammatory cell infiltration in patients with AIH is largely unknown [10]. Regardless of the initial trigger(s), it is likely that the high number of activated inflammatory cells seen in the periphery or in the liver may have the potential to cause damage in susceptible individuals [10].

Although it would be attractive to explain the pathogenesis of AIH by a single mechanism, it is now clear that several different mechanisms and pathological processes could break tolerance, thus terminating a previously unresponsive state to liver-related autoantigens. More than one defect may be present in AIH, and the defects may vary between type- 1 and type-2 AIH and among individuals affected by the same type of the disease. This approach may well explain several important principles of relevance to AIH and in particular its multi-factorial nature involving diverse immunological, genetic and environmental factors and the tendency of the disease to remit and relapse despite drug-induced immunosuppression, indicating that control mechanisms may recover and at least temporarily restore tolerance. Long periods of subclinical disease are often seen and many patients with acute presentation have histological evidence of chronic disease further suggesting that control mechanisms may continue to work up to a point.

Tolerance to liver-related antigens may be disrupted by several mechanisms $[10,12]$. The thesis that regulatory $\mathrm{T}$ cells that can limit the function of autoreactive T-cells are impaired in patients with AIH and may lead to the maintenance of disease-specific autoaggressive responses is quite attractive. In fact, early studies investigating immune regulation in patients with AIH have clearly demonstrated that the suppressor cell function is impaired in newly-diagnosed AIH patients [13-16]. These studies have also shown that corticosteroid treatment can properly restore immune regulation to liver-specific antigens [17]. Recent experimental evidence suggests that patients with AIH are characterized by a numerical and functional impairment of CD4+ CD25+ regulatory cells; such a defect relates to the histological stage of liver disease and is more evident at disease presentation than during treatment induced remission, where a partial restoration is observed $[18,19]$.

More recent studies have linked impaired apoptotic death to liver autoimmunity [20-22]. Thus, peripheral CD4 and CD8 T-cells expressing CD95 (Fas/APO-1) appear to be more prevalent in patients with AIH compared to pathological controls [20]. Over-expression of bcl-2 in peripheral and liver-infiltrating interferon- $\gamma$ CD4 T-cells has also been described in patients with AIH compared to controls [21]. Bcl2 expressing CD4 T-cells infiltrate the portal area of the liver and their numbers correlate with elevation of ALT levels at the time of disease relapse [22].

Others have failed to obtain evidence in support of a significant role for apoptosis in the pathogenesis of AIH. An immunohistochemical study by De Biasio et al. [23], for example, has reported that Fas-L and Bak apoptotic markers are uniformly distributed through the portal and periportal areas in liver biopsy specimens from patients with AIH-1 and chronic hepatitis $\mathrm{C}$ virus infected patients.

A role for viruses as triggers of AIH has been proposed but never proven because the infections responsible for the induction of the disease are likely to occur years before the onset of AIH [10, 24-26]. Moreover, the virus responsible for the breakdown of tolerance can be cleared by the host's immune system and may cannot be traced when the autoimmune process is initiated [26]. The exact mechanism by which viruses may participate in the immunopathogenesis of AIH is unclear [10, 26, 27].

It has been demonstrated in the past that molecular mimicry between pathogen and host structures may be involved in the induction, acceleration and prevention of autoimmune processes [28-33]. Along these lines, molecular mimicry involving viral and self structures has been suggested as a mechanism responsible for the loss of immunological tolerance in AIH but definite proof of this mechanism has been elusive in humans [26, 34-39]. Nevertheless, a considerable amount of data has been obtained suggesting that infection with viruses such as hepatitis $\mathrm{C}$, cytomegalovirus and herpes simplex virus may indeed initiate cross-reactive immune responses at B-cell and/or T-cell (CD4 and CD8) level involving mimicking autoepitopic regions on members of the cytochrome P450 including CYP2D6, the molecular target of AIH-2 specific LKM-1 autoantibodies [34-36, 38]. Such crossreactivities have specifically been found in patients with AIH or LKM-1 positive patients with chronic hepatitis C. Their pathogenic relevance is still unclear as immunological crossreactivity has been studied at the time of overt disease and therefore may just represent a 'footprint' of an ongoing process that took place several years before the onset of the full-blown clinical picture. Hence, the possibility that the observed viral/self cross-reactive responses seen in patients with AIH are secondary to the immunological breakdown of the disease cannot be neglected. Definite proof for the role of molecular mimicry as an initiator or perpetuator of autoimmunity will remain difficult to obtain in humans. These obstacles have led to increasing interest in animal models of AIH. Systematic studies of these models may provide new insights into the etiological factors and the pathogenetic mechanism of AIH. This review updates on current animal models for acute and chronic autoimmune-mediated hepatitis and their relevance to human $\mathrm{AIH}$.

\section{ANIMAL MODELS FOR HUMAN AUTOIMMUNE HEPATITIS}

Unfortunately, there is no primate model for human autoimmune hepatitis available to date. Below, we discuss models of autoimmune injury of the hepatic parenchyma with some similarities with the clinical, serological and histological features of human AIH. With the exception of the models focusing on breaking tolerance to CYP2D6, the target antigen of AIH-2, these models do not pay attention to a certain 
AIH subtype. The vast majority of models use wildtype or transgenic mice, in which liver damage is induced by various means of inflammatory insults, such as virus infection or injection of unspecific activators of the immune system. Often hepatitis was reported to be only transient and most models for autoimmune liver disease depend on a rather complex disease-induction protocol. Here, we will reflect on past models for AIH and introduce some of the newer model systems that have been developed over the last few years. Whereas for some autoimmune diseases, such as type 1 diabetes, suitable spontaneous models exist, such as the nonobese diabetic (NOD) mouse [40], there has only been one study to date that focused on the occurrence of spontaneous autoimmune liver damage in mice [41]. The male F1 hybrid mice resulting from mating NZW $x$ BXSB with W/BF1 mice showed spontaneous cellular infiltration of the portal tracts and the liver parenchyma with 20 weeks of age, developed anti-dsDNA antibodies and circulating immune complexes and had elevated serum aminotransferase levels [41]. Further studies using these mice have not been reported.

Most animal models for AIH that have been developed in the past two decades make use of transgenic mice, which express target antigens under the control of liver-specific promoters. Such a liver-specific expression ensures the initiation of an autoaggressive immune response specifically targeting the liver. However, even before the widespread use of transgenic technology, several models for AIH have been developed that used fractions of liver homogenates combined with polyclonal activators, such as complete Freund's adjuvant (cFA). Although the target antigens of human AIH were not entirely characterized at that time, some of those rather simple strategies resulted in persistent liver damage, an important feature, which (disturbingly) is lacking in many of the newer, more complex experimental models of AIH. In the 70's, it was demonstrated that repetitive immunization of rabbits with unidentified human liver-specific lipoprotein resulted in persistent hepatitis [2]. One of the earliest mouse models was termed 'experimental autoimmune hepatitis' or 'EAH' [42] in analogy to experimental autoimmune encephalomyelitis (EAE), which was at that time already widely used as a model for human multiple sclerosis (MS) [43]. Lohse et al. induced EAH by immunization of C57BL/6 mice with a crude $100,000 \mathrm{~g}$ supernatant of syngeneic liver homogenate (S-100) emulsified in complete Freund's adjuvant (cFA) [44]. They demonstrated a long lasting (at least 6 months) liver damage characterized by perivascular inflammatory infiltrates and hepatocyte necrosis and further identified S-100 protein-specific T-cells [44]. This model is still in use, and a recent study based on this model demonstrated that the mitogen-activated protein kinase (MAPK) p38 signaling pathway is up-regulated in EAH. Inhibition of p38 MAPK reduced hepatic inflammation and injury possibly by decreasing NF- $\kappa B$ activation and Th1 cytokine expression and Th1 cytokines (IFN- $\gamma$, IL-12, IL-1 $\beta$ and TNF- $\alpha$ ) known to promote hepatic injury in AIH [45].

A widely used method to induce acute liver inflammation is the injection of Concanavalin A (ConA) [46], which accumulates in the liver [47] and causes liver damage by activating T-cells and NKT cells that subsequently destroy hepatocytes in a FasL and perforin-dependent manner [9, 48]. However, ConA-induced hepatitis is a model for acute hepatic injury characterized by a massive elevation of serum aminotransferase levels within hours of ConA administration, caused by the sheer magnitude of hepatocyte death by cell lysis and apoptosis. Although ConA hepatitis is not a true model for chronic autoimmune-mediated liver damage as seen in human $\mathrm{AIH}$, it serves as an excellent and simple model to investigate acute T-cell and NKT activation and their associated inflammatory factors. Very recently, for example, Halder et al. demonstrated that activation of type II NKT cells and plasmacytoid DCs recruit type I NKT (iNKT) cells into the liver in a IL-12- and MIP-2-dependent manner [49]. Such recruited iNKT cells were anergic and prevented ConA-induced hepatitis [49].

\section{Transgenic Models}

The advantage of using transgenic mice lays in the presence of a clearly-defined antigen as a target for the aggressive immune response. In addition, tissue-specific expression of the target antigen allows directing the autoimmunemediated damage to the tissue/organ of choice. However, the presence of the transgenic target antigen in most cases induces an inherent tolerance of the host. Thus, in order to break this tolerance several approaches have been chosen. A first possibility is to induce a massive activation of the immune system by administration of adjuvant or stimulators of the innate response, such as LPS. Second, the inherent tolerance mechanism can be circumvented by transferring target antigen-specific T-cells (mostly of TcR-transgenic origin). Lastly, virus-infection targeting the liver can cause a local 'fertile field' [50] resulting in enhanced attraction of potential aggressive cells of the immune system.

In the early 1990s, Frank Chisari's group generated transgenic mice expressing specifically in hepatocytes the hepatitis B virus surface antigen (HBsAg) under the control of the mouse albumin promotor. In this model, an adoptive transfer of activated T-cells from an HBsAg-primed donor mouse was necessary to induce disease [51, 52]. It was found that the $\mathrm{HBsAg}$-specific immune response was dominated by HBsAg-specific cytotoxic T-lymphocytes (CTL) that triggered apoptosis of hepatocytes expressing $\mathrm{HBsAg}$ and released IFN $\gamma$ upon antigen encounter [51]. As a consequence, intrahepatic macrophages became activated and subsequently induced a delayed-type hypersensitivity reaction [51]. However, only a transient form of hepatic injury was observed which lasted less than 3 weeks following the transfer of activated HBsAg-specific cells [51].

In a subsequent model, the MHC class I molecule $\mathrm{H}-2 \mathrm{~K}^{\mathrm{b}}$ was expressed directly in the liver of TcR-transgenic mice carrying for $\mathrm{H}-2 \mathrm{~K}^{\mathrm{b}}$-specific $\mathrm{T}$-cells that display peripheral tolerance to $\mathrm{H}-2 \mathrm{~K}^{\mathrm{b}}$ [53]. In that system, tolerance could only be broken after additional transfer of cells expressing the $\mathrm{H}$ $2 \mathrm{~K}^{\mathrm{b}}$ target antigen and IL-2, or alternatively when mice were infected with a liver-specific pathogen, indicating that bystander activation within the liver microenvironment is necessary to cause autoimmune tissue damage [53]. In a follow up paper by the same authors it was demonstrated that injection of immunostimulatory CpG-rich oligodeoxynucleotides (CpG-ODN) in $\mathrm{H}-2 \mathrm{~K}^{\mathrm{b}} / \mathrm{TcR}$-doubletransgenic mice is sufficient to activate $\mathrm{H}-2 \mathrm{~K}^{\mathrm{b}}$-specific CD8 T-cells, which then subsequently attack $\mathrm{H}-2 \mathrm{~K}^{\mathrm{b}}$-expressing hepatocytes [54]. However, in order to maintain hepatic damage over a longer period of time ( $>8$ weeks) CpG-ODN had to be repeatedly 
Table 1. Mouse Models for Human Autoimmune Hepatitis

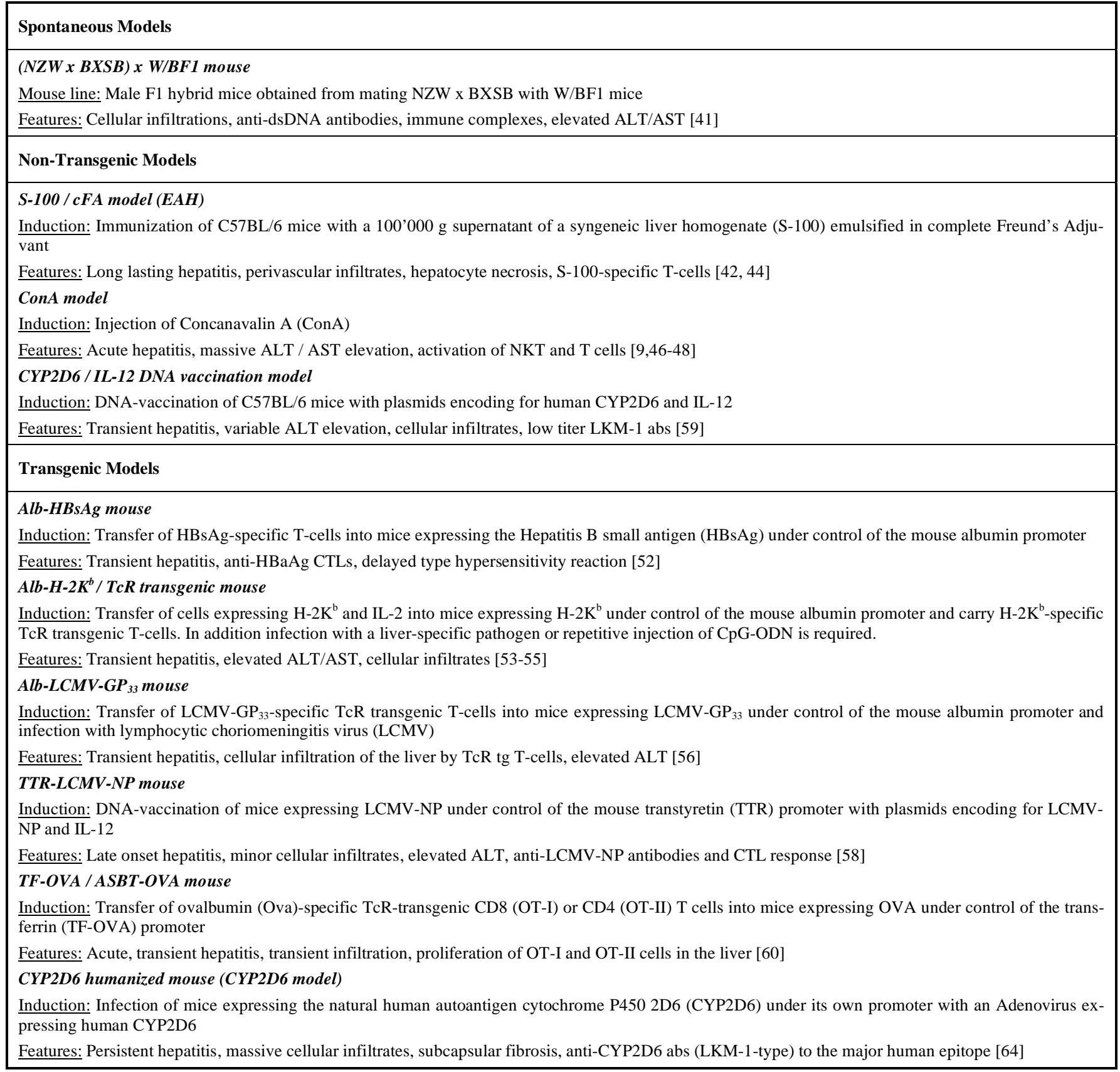

administrated and termination of this inflammatory stimulus resulted in abrogation of disease. These findings indicated that additional factors are required for the self-perturbation of autoimmunity [54]. In this context, Bertolini et al. reported the appearance of adoptively transferred $\mathrm{H}-2 \mathrm{~K}^{\mathrm{b}}$ specific $\mathrm{T}$-cells in the liver of $\mathrm{H}-2 \mathrm{~K}^{\mathrm{b}}$-transgenic mice as early as 2 hours after injection [55].

Voehringer et al. demonstrated that breaking of T-cell ignorance to a viral (transgene) antigen in the liver could induce hepatitis [56]. They used transgenic mice that expressed the immunodominant CTL epitope $\mathrm{GP}_{33}$ of the glycoprotein (GP) of the lymphocytic choriomeningitis virus (LCMV) under the control of the albumin promotor exclusively in the liver. Such Alb-LCMV mice did not show any signs of hepatitis at that stage; even adoptive transfer of
TcR-transgenic, $\mathrm{GP}_{33}$-specific T-cells was not sufficient to induce disease. However, when these mice were infected with LCMV after adoptive transfer of TcR-tg cells, a transient form of hepatitis developed [56]. This approach is similar to the RIP-LCMV mouse model for type 1 diabetes, where GP is expressed under control of the rat insulin promotor (RIP) specifically in the insulin-producing $\beta$-cells in the pancreas [57]. However, in contrast to the Alb-LCMV model, the classical RIP-LCMV model does not require transfer of LCMV-specific T-cells [57].

Another model system, which used one of the wellcharacterized LCMV proteins as targets, is the TTR-LCMV mouse expressing LCMV-NP under the control of the transtyretin (TTR) promotor specifically in the liver [58]. Liver injury characterized by cellular infiltration and elevated se- 
rum aminotransferase levels was induced by DNAvaccination with plasmids encoding the NP and IL-12 genes. An NP-specific CTL response was detectable after 2 months and persisted up to 5 months post-vaccination [58]. However, serum aminotransferase levels were not increased before month 5 and only minor cellular infiltrates were detectable in the liver [58].

In a subsequent study from Alvarez group, an attempt was made to induce autoimmune liver damage by xenoimmunization using a plasmid containing the antigenic region of CYP2D6 [59]. However, only co-expression of the Th1type cytokine IL-12 together with CYP2D6 resulted in significant inflammation in the liver. In addition, only minor elevations in serum ALT and a transient generation of antiCYP2D6 antibodies with large inter-individual differences were stated [59].

More recently, Derkow et al. generated transgenic TFOVA and ASBT-OVA mice, which express the model antigen ovalbumin (OVA) under control of the transferring promotor (TF) or the apical sodium-dependent bile transporter promotor (ASBT) specifically in hepatocytes or cholangiocytes, respectively [60]. The authors adoptively transferred TCR-transgenic CD8 T-cells (OT-I) or CD4 T-cells (OT-II) and found that only OT-I T-cells migrated to the liver in TFOVA as well as ASBT-OVA mice, where they proliferated and caused inflammation and acute liver injury [60]. However, the elevation of serum aminotransferases and cellular infiltration lasted only for 1-2 weeks post-transfer [60].

\section{The CYP2D6 Mouse Model}

The models discussed above demonstrate that induction of chronic autoimmunity targeting specifically the liver is a difficult task. In most models, the liver injury is transient and can only be achieved by rather complex intervention methods. Often, tolerance to the transgenic target antigen can only be broken when antigen-specific T-cells are transferred and additional inflammatory triggers are used, such as virus infections or overexpression of pro-inflammatory cytokines. There are several possibilities why most model systems result in acute hepatitis only. First, the liver appears somewhat resistant to long-lasting autoimmune-mediated damage due to local mechanism involving $\mathrm{T}$ cell deletion, inactivation and apoptosis. Thus, an initial acute liver injury (i.e. by virus infection) might not necessarily lead to autoimmunity. Second, models that require both the presence of a transgenic target antigen and the transfer of target antigen-specific TcRtransgenic $\mathrm{T}$ cells might encounter the problem of $\mathrm{T}$ cell exhaustion. Therefore, a continuous transfer of TcR-transgenic $\mathrm{T}$ cells might be required to maintain a chronic destruction process. Third, models that require the additional expression of pro-inflammatory cytokines might induce counteracting mechanisms to balance out the pro-inflammatory milieu in the liver. Last, most models do not use a natural human autoantigen as a target for the autoimmune liver destruction but rather an artificial model antigen which may not be sufficient for inducing chronic disease.

Our strategy was therefore to use a natural human autoantigen as a target. We used human CYP2D6, which constitutes the major target autoantigen in human type-2 AIH [61, 62]. In humanized CYP2D6 mice, the human CYP2D6 is transgenically expressed under control of its own promotor predominantly in the liver [63]. Natural tolerance to this autoantigen was broken by infection of CYP2D6 mice with an Adenovirus-vector expressing human CYP2D6 (Ad2D6). We observed long lasting hepatic damage, characterized by massive subcapsular liver fibrosis, extensive subcapsular and peri-vascular infiltration by B-cells, CD4 and CD8 T-cells, macrophages and dendritic cells, and transiently elevated serum aminotransferase levels [64]. Importantly, Ad-2D6-infected CYP2D6 mice develop high titers of antiCYP2D6 antibodies and recognize the same immunodominant epitope which is recognized by AIH-2 patients [64]. An overview of the liver damage seen overtime in CYP2D6 mice after infection with Ad-2D6 or Ad-GFP is displayed schematically in Fig. (2). Furthermore, infection of CYP2D6 mice with Ad-CYP2D6 causes an activation of hepatic stellate cells (HSC) and subsequently persistent fibrosis, which is localized predominantly in the subcapsular area (Hintermann and Christen, unpublished observation). The CYP2D6mouse may therefore represent a valid model system to investigate mechanisms involved in the immunopathogenesis of autoimmune mediated chronic hepatic injury as seen in human $\mathrm{AIH}$ and to evaluate possible ways of therapeutic interference.

\section{Liver damage in the CYP2D6 mouse model}
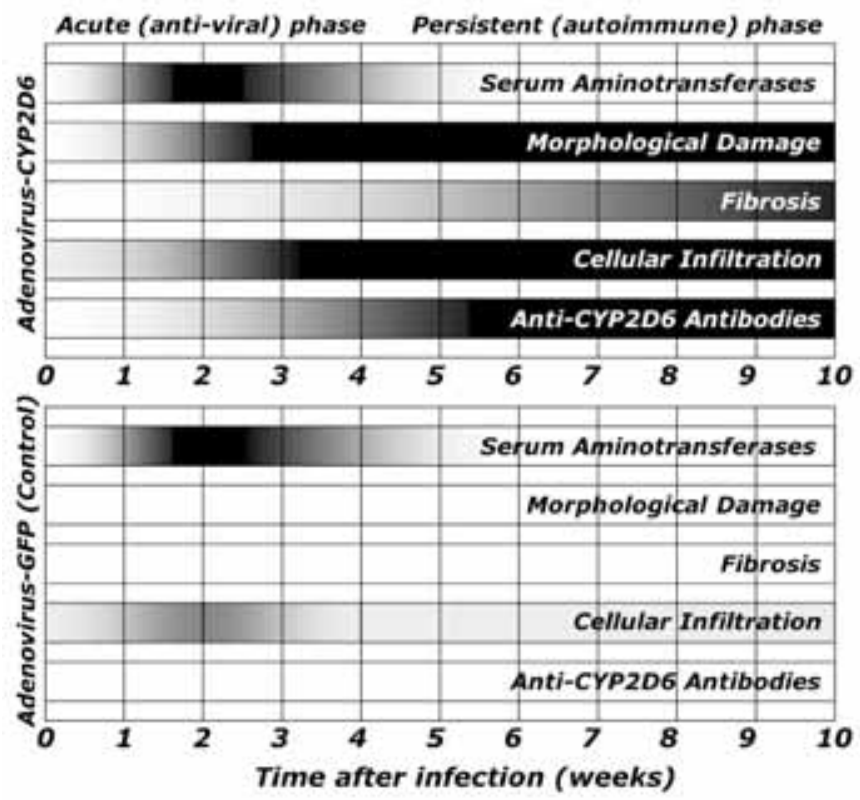

Fig. (2). Summary of the main immunopathological characteristics of the humanized cytochrome P450 2D6 (CYP2D6) mouse model. Biochemical evidence of liver damage (elevation of serum aminotransferases), overall morphological damage, fibrosis, cellular infiltration and the generation of anti-CYP2D6 antibodies are displayed over time after infection of CYP2D6 mice with either Adenovirus-CYP2D6 or the control Adenovirus expressing GFP (Highest effects are displayed in black, lowest effects in white). Note that an acute (anti-viral) response occurs after infection with either Adenovirus-CYP2D6 or GFP, whereas only Adenovirus-CYP2D6infection resulted in a persistent (autoimmune) phase.

As it has mentioned earlier, human AIH is a chronic autoimmune liver disease resulting in the progressive destruction of the liver parenchyma. Thus, it is important to distinguish animal models associated with acute liver injury from those developing chronic active hepatitis. Each model 
has certain limitations and can only help us to appreciate certain aspects of the complex immunopathology of AIH. None of the models presented above cover all the aspects of human AIH. Thus, one has to consider all the characteristics of the model of choice in order to make a reliable extrapolation to the human disease. Models of acute liver-induced immune-mediated injury, such as ConA-induced hepatitis cover aspects of immediate inflammation and initial activation of the innate immune system and thus are well suited for identifying critical inflammatory factors such as cytokines and chemokines. Blockade of such factors might offer an opportunity for preventing the initial activation of autoaggression resulting in hepatocyte destruction. On the other hand, chronic models can advance our knowledge of longlasting mechanisms leading to liver pathology. They can help us to identify the initial pathogenic insult and to understand the hierarchy of immunological events. The humanized CYP2D6 model of AIH, for example, can help us to critically analyze disease-specific B- and T-cell immune responses against a liver autoantigen and the relation of immunity to the development of liver fibrosis $[64,65]$. In this context, the CYP2D6 mouse model is superior to others as it combines an acute hepatitis phase caused by the Adenovirusinfection per se with a chronic immune-mediated hepatitis as a result of the progressive autoimmune-mediated liver injury. Thus, the CYP2D6 model may offer a unique opportunity to study all mechanisms of virus-induced autoimmunity ranging from the initial activation of the innate immune system to the autoimmune-mediated destruction of the liver.

\section{ABBREVIATIONS}

$$
\begin{aligned}
\text { AIH } & \text { Autoimmune hepatitis } \\
\text { LKM-1 } & \text { Liver kidney microsomal type } 1 \\
\text { CYP2D6 } & \text { Cytochrome P450 2D6 } \\
\text { Ad-2D6 } & \text { Adenovirus-vector expressing human } \\
& \text { CYP2D6 }
\end{aligned}
$$

\section{ACKNOWLEDGEMENT}

We thank Professor Diego Vergani for critical reading and helpful comments.

\section{REFERENCES}

[1] Krawitt EL. Autoimmune hepatitis. N Engl J Med 2006; 354(1): 54-66.

[2] Alvarez F, Berg PA, Bianchi FB, et al. International Autoimmune Hepatitis Group Report: review of criteria for diagnosis of autoimmune hepatitis. J Hepatol 1999; 31(5): 929-38.

[3] Boberg KM. Prevalence and epidemiology of autoimmune hepatitis. Clin Liver Dis 2002; 6(3): 635-47.

[4] Czaja AJ. Autoimmune hepatitis-approach to diagnosis. Med Gen Med 2006; 8(2): 55.

[5] Gregorio GV, Portmann B, Reid F, et al. Autoimmune hepatitis in childhood: a 20-year experience. Hepatology 1997; 25(3): 541-7.

[6] Vergani D, Alvarez F, Bianchi FB, et al. Liver autoimmune serology: a consensus statement from the committee for autoimmune serology of the International Autoimmune Hepatitis Group. J Hepatol 2004; 41(4): 677-83.

[7] Czaja AJ, Homburger HA. Autoantibodies in liver disease. Gastroenterology 2001; 120(1): 239-49.

[8] Liaskos C, Bogdanos DP, Davies ET, Dalekos GN. Diagnostic relevance of anti-filamentous actin antibodies in autoimmune hepatitis. J Clin Pathol 2007; 60(1): 107-8.

[9] Dennert G, Aswad F. The role of NKT cells in animal models of autoimmune hepatitis. Crit Rev Immunol 2006; 26(5): 453-73.
[10] Vergani D, Choudhuri K, Bogdanos DP, Mieli-Vergani G. Pathogenesis of autoimmune hepatitis. Clin Liver Dis 2002; 6(3): 727 37.

[11] Vergani D, Mieli-Vergani G. Autoimmune hepatitis. Autoimmun Rev 2003; 2(5): 241-7.

[12] Kita H, Mackay IR, Van De Water J, Gershwin ME. The lymphoid liver: considerations on pathways to autoimmune injury. Gastroenterology 2001; 120(6): 1485-501.

[13] Nouri-Aria KT, Donaldson PT, Hegarty JE, Eddleston AL, Williams R. HLA A1-B8-DR3 and suppressor cell function in firstdegree relatives of patients with autoimmune chronic active hepatitis. J Hepatol 1985; 1(3): 235-41.

[14] Nouri-Aria KT, Lobo-Yeo A, Vergani D, Mieli-Vergani G, Eddleston AL, Mowat AP. T suppressor cell function and number in children with liver disease. Clin Exp Immunol 1985; 61(2): 283-9.

[15] Vento S, O'Brien CJ, McFarlane IG, Williams R, Eddleston AL. Tcell inducers of suppressor lymphocytes control liver-directed autoreactivity. Lancet 1987; 1(8538): 886-8.

[16] Vento S, Hegarty JE, Bottazzo G, Macchia E, Williams R, Eddleston AL. Antigen specific suppressor cell function in autoimmune chronic active hepatitis. Lancet 1984; 1(8388):1200-4.

[17] Nouri-Aria KT, Hegarty JE, Alexander GJ, Eddleston AL, Williams R. Effect of corticosteroids on suppressor-cell activity in "autoimmune" and viral chronic active hepatitis. N Engl J Med 1982; 307(21): 1301-4.

[18] Longhi MS, Ma Y, Bogdanos DP, Cheeseman P, Mieli-Vergani G, Vergani D. Impairment of CD4(+) CD25(+) regulatory T-cells in autoimmune liver disease. J Hepatol 2004; 41(1): 31-7.

[19] Longhi MS, Ma Y, Mitry RR, et al. Effect of CD4+ CD25+ regulatory T-cells on CD8 T-cell function in patients with autoimmune hepatitis. J Autoimmun 2005; 25(1): 63-71.

[20] Ogawa S, Sakaguchi K, Takaki A, et al. Increase in CD95 (Fas/APO-1)-positive CD4+ and CD8+ T cells in peripheral blood derived from patients with autoimmune hepatitis or chronic hepatitis C with autoimmune phenomena. J Gastroenterol Hepatol 2000; 15(1): 69-75.

[21] Yachida M, Kurokohchi K, Arima K, Nishioka M. Increased bcl-2 expression in lymphocytes and its association with hepatocellular damage in patients with autoimmune hepatitis. Clin Exp Immunol 1999; 116(1): 140-5.

[22] Kurokohchi K, Arima K, Masaki T, et al. Analysis of CD28 and bcl-2 expression on peripheral blood and liver-infiltrating mononuclear cells in patients with autoimmune hepatitis. J Clin Immunol 2006; 26(4): 323-30.

[23] De Biasio MB, Periolo N, Avagnina A, et al. Liver infiltrating mononuclear cells in children with type 1 autoimmune hepatitis. J Clin Pathol 2006; 59(4): 417-23.

[24] Vento S, Cainelli F. Is there a role for viruses in triggering autoimmune hepatitis? Autoimmun Rev 2004; 3(1): 61-9.

[25] Bogdanos DP, Mieli-Vergani G, Vergani D. Virus, liver and autoimmunity. Dig Liver Dis 2000; 32(5): 440-6.

[26] Bogdanos DP, Choudhuri K, Vergani D. Molecular mimicry and autoimmune liver disease: virtuous intentions, malign consequences. Liver 2001; 21(4): 225-32.

[27] Christen U, von Herrath MG. Infections and autoimmunity-good or bad? J Immunol 2005; 174(12): 7481-6.

[28] Rose NR, Mackay IR. Molecular mimicry: a critical look at exemplary instances in human diseases. Cell Mol Life Sci 2000; 57(4): 542-51.

[29] Oldstone MB. Molecular mimicry and immune-mediated diseases. FASEB J 1998; 12(13): 1255-65.

[30] Fujinami RS, von Herrath MG, Christen U, Whitton JL. Molecular mimicry, bystander activation, or viral persistence: infections and autoimmune disease. Clin Microbiol Rev 2006; 19(1): 80-94.

[31] Christen U, Edelmann KH, McGavern DB, et al. A viral epitope that mimics a self antigen can accelerate but not initiate autoimmune diabetes. J Clin Invest 2004;114(9):1290-8.

[32] Christen U, von Herrath MG. Induction, acceleration or prevention of autoimmunity by molecular mimicry. Mol Immunol 2004; 40(14-15): 1113-20.

[33] Croxford JL, Anger HA, Miller SD. Viral delivery of an epitope from Haemophilus influenzae induces central nervous system autoimmune disease by molecular mimicry. J Immunol 2005; 174(2): 907-17. 
[34] Manns MP, Griffin KJ, Sullivan KF, Johnson EF. LKM-1 autoantibodies recognize a short linear sequence in P450IID6, a cytochrome P-450 monooxygenase. J Clin Invest 1991; 88(4): 1370-8.

[35] Kammer AR, van der Burg SH, Grabscheid B, et al. Molecular mimicry of human cytochrome $\mathrm{P} 450$ by hepatitis $\mathrm{C}$ virus at the level of cytotoxic T cell recognition. J Exp Med 1999; 190(2): 16976.

[36] Kerkar N, Choudhuri K, Ma Y, et al. Cytochrome P4502D6 (193212): a new immunodominant epitope and target of virus/self cross-reactivity in liver kidney microsomal autoantibody type 1positive liver disease. J Immunol 2003; 170(3): 1481-9.

[37] Bogdanos DP, Smith H, Ma Y, Baum H, Mieli-Vergani G, Vergani D. A study of molecular mimicry and immunological crossreactivity between hepatitis $\mathrm{B}$ surface antigen and myelin mimics. Clin Dev Immunol 2005; 12(3): 217-24.

[38] Bogdanos DP, Lenzi M, Okamoto M, et al. Multiple viral/self immunological cross-reactivity in liver kidney microsomal antibody positive hepatitis $\mathrm{C}$ virus infected patients is associated with the possession of HLA B51. Int J Immunopathol Pharmacol 2004; 17(1): 83-92.

[39] Bogdanos DP, Rigopoulou EI. Viral/self-mimicry and immunological cross-reactivity as a trigger of hepatic $\mathrm{C}$ virus associated autoimmune diabetes. Diabetes Res Clin Pract 2007; 77(1): 155-6.

[40] Delovitch TL, Singh B. The nonobese diabetic mouse as a model of autoimmune diabetes: Immune dysregulation gets the NOD. Immunity 1997; 7: 727-38.

[41] Amoh Y, Yasumizu R, Yamamoto Y, et al. The appearance of unusual phenotypic cells (CD4+ Mac-1+ class II+) in the liver of (NZW x BXSB)F1 mice is possibly an animal model for autoimmune hepatitis. Immunobiology 1997; 197(1): 31-43.

[42] Kuriki J, Murakami H, Kakumu S, et al. Experimental autoimmune hepatitis in mice after immunization with syngeneic liver proteins together with the polysaccharide of Klebsiella pneumoniae. Gastroenterology 1983; 84(3): 596-603.

[43] Baxter AG. The origin and application of experimental autoimmune encephalomyelitis. Nat Rev Immunol 2007; 7(11): 904-12.

[44] Lohse AW, Manns M, Dienes HP, Meyer zum Buschenfelde KH, Cohen IR. Experimental autoimmune hepatitis: disease induction, time course and T-cell reactivity. Hepatology. 1990; 11(1): 24-30.

[45] Ma X, Jia YT, Qiu DK. Inhibition of p38 mitogen-activated protein kinase attenuates experimental autoimmune hepatitis: involvement of nuclear factor kappa B. World J Gastroenterol 2007; 13(31): 4249-54.

[46] Tiegs G, Hentschel J, Wendel A. A T cell-dependent experimental liver injury in mice inducible by concanavalin A. J Clin Invest 1992; 90(1): 196-203.

[47] Gantner F, Leist M, Lohse AW, Germann PG, Tiegs G. Concanavalin A-induced T-cell-mediated hepatic injury in mice: the role of tumor necrosis factor. Hepatology 1995; 21(1): 190-8.

[48] Takeda K, Hayakawa Y, Van Kaer L, Matsuda H, Yagita H, Okumura K. Critical contribution of liver natural killer $\mathrm{T}$ cells to a murine model of hepatitis. Proc Natl Acad Sci USA 2000; 97(10): 5498-503.

[49] Halder RC, Aguilera C, Maricic I, Kumar V. Type II NKT cellmediated anergy induction in type I NKT cells prevents inflammatory liver disease. J Clin Invest 2007; 117(8): 2302-12.
[50] von Herrath MG, Fujinami RS, Whitton JL. Microorganisms and autoimmunity: making the barren field fertile. Nat Rev Microbiol 2003; 1: 151-7.

[51] Christen U, Quinn J, Yeaman SJ, et al. Identification of the dihydrolipoamide acetyltransferase subunit of the human pyruvate dehydrogenase complex as an autoantigen in halothane hepatitis. Molecular mimicry of trifluoroacetyl-lysine by lipoic acid. Eur J Biochem 1994; 223(3): 1035-47.

[52] Moriyama T, Guilhot S, Klopchin K, et al. Immunobiology and pathogenesis of hepatocellular injury in hepatitis B virus transgenic mice. Science 1990; 248(4953): 361-4.

[53] Limmer A, Sacher T, Alferink J, et al. Failure to induce organspecific autoimmunity by breaking of tolerance: importance of the microenvironment. Eur J Immunol 1998; 28(8): 2395-406.

[54] Sacher T, Knolle P, Nichterlein T, Arnold B, Hammerling GJ, Limmer A. CpG-ODN-induced inflammation is sufficient to cause T-cell-mediated autoaggression against hepatocytes. Eur J Immunol 2002; 32(12): 3628-37.

[55] Bertolino P, Bowen DG, McCaughan GW, Fazekas de St Groth B. Antigen-specific primary activation of CD8+ $\mathrm{T}$ cells within the liver. J Immunol 2001; 166(9): 5430-8.

[56] Voehringer D, Blaser C, Grawitz AB, Chisari FV, Buerki K, Pircher $\mathrm{H}$. Break of $\mathrm{T}$ cell ignorance to a viral antigen in the liver induces hepatitis. J Immunol 2000; 165(5): 2415-22.

[57] Fujinami RS, Oldstone MB. Amino acid homology between the encephalitogenic site of myelin basic protein and virus: mechanism for autoimmunity. Science 1985; 230(4729): 1043-5.

[58] Djilali-Saiah I, Lapierre P, Vittozi S, Alvarez F. DNA vaccination breaks tolerance for a neo-self antigen in liver: a transgenic murine model of autoimmune hepatitis. J Immunol 2002; 169(9): 4889-96.

[59] Lapierre P, Djilali-Saiah I, Vitozzi S, Alvarez F. A murine model of type 2 autoimmune hepatitis: Xenoimmunization with human antigens. Hepatology 2004; 39(4): 1066-74.

[60] Derkow K, Loddenkemper C, Mintern J, et al. Differential priming of CD8 and CD4 T-cells in animal models of autoimmune hepatitis and cholangitis. Hepatology 2007; 46(4): 1155-65.

[61] Manns MP, Johnson EF, Griffin KJ, Tan EM, Sullivan KF. Major antigen of liver kidney microsomal autoantibodies in idiopathic autoimmune hepatitis is cytochrome P450db1. J Clin Invest 1989; 83(3): 1066-72.

[62] Zanger UM, Hauri HP, Loeper J, Homberg JC, Meyer UA. Antibodies against human cytochrome P-450db1 in autoimmune hepatitis type II. Proc Natl Acad Sci USA 1988; 85(21): 8256-60.

[63] Corchero J, Granvil CP, Akiyama TE, et al. The CYP2D6 Humanized Mouse: Effect of the Human CYP2D6 Transgene and HNF4alpha on the Disposition of Debrisoquine in the Mouse. Mol Pharmacol 2001; 60(6): 1260-7.

[64] Holdener M, Hintermann E, Bayer M, et al. Breaking tolerance to the natural human liver autoantigen Cytochrome P450 2D6 by virus infection. J Exp Med (in press).

[65] Longhi MS, Hussain MJ, Bogdanos DP, et al. Cytochrome P450IID6-specific CD8 T cell immune responses mirror disease activity in autoimmune hepatitis type 2. Hepatology 2007; 46(2): 472-84.

[66] Christen U, Holdener M, Hintermann E. Animal models for autoimmune hepatitis. Autoimmun Rev 2007; 6(5): 306-11. 